CLAWAR 2020: $23^{\text {rd }}$ International Conference on

Climbing and Walking Robots and the Support

Technologies for Mobile Machines,

Moscow, Russian Federation, 24-26 August 2020.

https://doi.org/10.13180/clawar.2020.24-26.08.02

\title{
DESIGN AND DEVELOPMENT OF A MULTI-ROBOT SYSTEM FOR BLOCKAGE REMOVAL
}

\author{
SERGEY MANKO, VALERY LOKHIN, SEKOU DIANE and VLADIMIR TSYPKIN \\ Control Problems Department, RTU MIREA, Vernadsky Ave 78, \\ Moscow, 119454, Russia \\ E-mail: cpd@mirea.ru,kpu-mirea@yandex.ru,sekoudiane1990@gmail.com,tsypkin@mirea.ru \\ www.mirea.ru
}

\begin{abstract}
Experimental model of a multi-robot system for full-scale testing of automatic blockage analysis and removal tasks is developed. The system is composed of autonomous with the appropriate set of software for planning movements of the mobile platform and the on-board manipulator using visual feedback. We describe software and algorithmic tools for scheduling and distributing subtasks, as well as coordinating movements of the robots. Software for the strategic level of group control allows determining the stages of operations for grasping and transporting individual elements of the blockage, which were detected during preliminary exploration. A knowledge base on the stages and specifics of technological operations allows for correct processing of information and making appropriate decisions in the process of multi-robot system operation. Proposed action planning algorithms are built on the basis of the finite state machine theory. Experimental studies have proved the reality of the prospects for creating multi-agent systems based on autonomous robots for solving complex problems in conditions of uncertainty.
\end{abstract}

\section{Introduction}

Currently the potential opportunities and advantages of using multi-agent robotic systems (MRS) for solving a number of applied tasks are obvious in the fields of exploration, construction, agriculture, utilities, etc. Combining several robotic agents in a group not only increases the speed and reliability of mission completion, but also reduces the cost of developing a technical solution by distributing functionality among several robotic agents in the group.

In general, the MRS control system designed to solve complex applications should include three main subsystems: action planning module, task distribution module and information processing subsystem. At the same time, the details of hardware and software implementation of these subsystems largely depend on the specifics of the applied problem being solved.

The problem of planning is one of the central ones in the field of artificial intelligence. In essence, planning is the process of optimally allocating resources to achieve goals, which is of particular interest in the field of multi-agent robotic systems.

There is a distinction between planning as determining assignments for agents in accordance with a known sequence of technological operations (scheduling) and planning under conditions of uncertainty of the scenario of the task being solved.

Thus, [1] presents an approach based on finite state machine (FSM) technology for mission planning of a group of autonomous underwater vehicles. In [2], an algorithm for dynamic scenario formation based on the analysis of sensory data is presented.

Planning can be carried out both at the level of MRS when determining the sequence of tasks and assigning assignments between agents, and at the level of individual agents, e.g. when searching for a route on the ground for a mobile robot.

The solution of planning problems is possible with the use of various technologies - graph search methods for linear and non-linear problems $[3,4]$ and universally applicable evolutionary algorithms [5]. 
Our work is based on a well-established finite state machine technology for solving scenario problems, but a significant difference in our approach is the consideration of complex MRS problems that require description in the form of hierarchically nested scenarios.

\section{Task planning}

Planning process has a hierarchical nature, going from scheduling tasks at the top level down to distributing tasks among the robots and finally to the fulfillment of tasks by individual agents (Fig. 1). This approach is applicable to a number of multi-stage applied problems solved using MRS, according to some a priori known or implicitly defined scenario of their fulfillment (including the construction of modular structures, debris removal, research and mapping of mazes, etc.).

Mathematical model of such problems can be formed as a network of typical finite state machines, each of which is related to a separate technological operation. This allows us to provide an adequate reflection of their fulfillment state and implement the process of continuous switching between the stages of the mission.

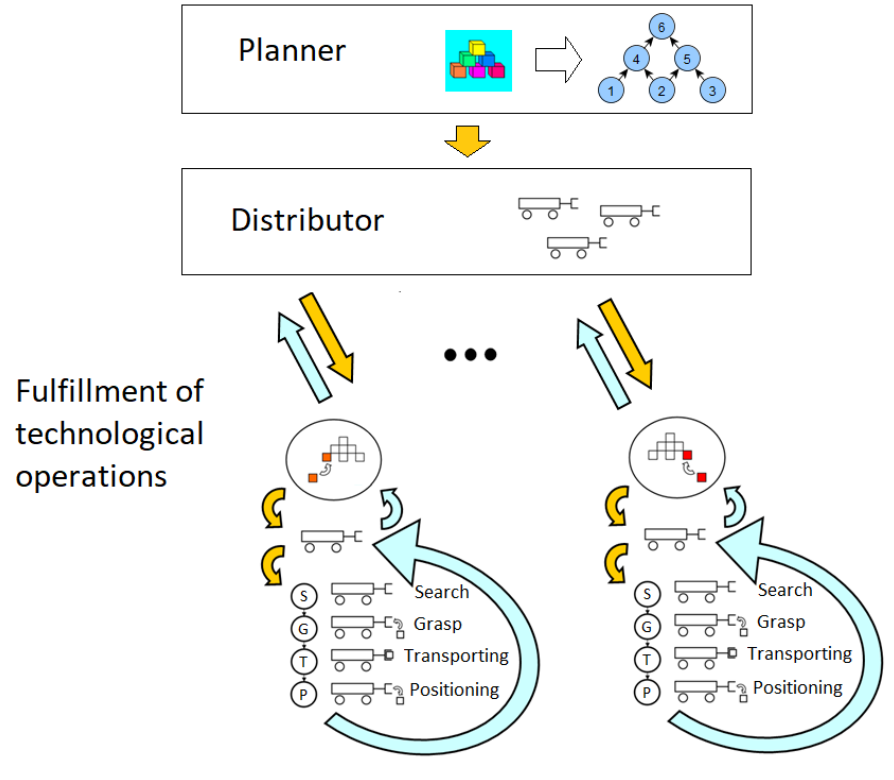

Figure 1. Hierarchical scheme of the planning process

A network of typical FSMs, each of which represents a single technological operation, can be formalized with a directed graph $G=\{V, D, S\}$, where $V=\left\{v_{i} / i=1,2, \ldots, n\right\}$ is a set of nodes in the graph; $D=\left\{t_{i}, p_{i}, K_{i}^{O}, G_{i} / i=1,2, \ldots, n\right\}$ is a set of data items associated with corresponding nodes, including task name $t_{i}$; task description $p_{i} ; \mathrm{FSM}$ variables $K_{i}^{O}$ and $G_{i}$ (an optional item keeping the node internal scenario); $S=\left\{s_{j} / j=1,2, \ldots, m\right\}$ is a set of edges in the graph.

Thus, graph edges determine the feasible execution sequence for a set of technological operations, and the states of the FSMs associated to the graph nodes reflect the execution stage of corresponding operations.

In this paper we continue an approach that we presented in [2] and use a typical FSM control element for modeling a technological operation in the following form: 


$$
K^{O}=\left\{U^{O}, X^{O}, Y^{O}, f^{O}, h^{O}\right\}
$$

where $U^{O}$ is an input alphabet; $X^{O}$ is an alphabet of task states; $Y^{O}$ is an output alphabet that confirms task current states; $f^{O}, h^{O}$ are state-transition and output functions of the FSM. All the listed elements are further clarified in the Table I.

Table 1. FSM model of a technological operation

\begin{tabular}{|l|c|c|c|c|}
\hline State & $\begin{array}{c}x_{0}^{O} \\
\text { (Blocked) }\end{array}$ & $\begin{array}{c}x_{1}^{O} \\
\text { (Pending) }\end{array}$ & $\begin{array}{c}x_{2}^{O} \\
\text { (In process) }\end{array}$ & $\begin{array}{c}x_{3}^{O} \\
\text { (Finished) }\end{array}$ \\
\hline$u_{0}^{O}$ (Blocked) & $x_{0}^{O}$ & $x_{1}^{O}$ & $x_{2}^{O}$ & $x_{3}^{O}$ \\
\hline$u_{1}^{O}$ (Pending) & $x_{1}^{O}$ & $x_{1}^{O}$ & $x_{2}^{O}$ & $x_{3}^{O}$ \\
\hline$u_{2}^{O}$ (In process) & $x_{2}^{O}$ & $x_{2}^{O}$ & $x_{2}^{O}$ & $x_{3}^{O}$ \\
\hline$u_{3}^{O}$ (Finished) & $x_{3}^{O}$ & $x_{3}^{O}$ & $x_{3}^{O}$ & $x_{3}^{O}$ \\
\hline
\end{tabular}

Using the above suggested model, the scenario fulfillment may be controlled through the detection of operations which are available for execution after completion of their predecessors. The complexity of the FSM model allows to formulate MRS task scheduling algorithm in the following simple steps:

1. Initialization of the list of the tasks available for the processing $L^{*}:=\varnothing$;

2. Initialization of the loop variable: $i:=0$;

3. If FSM $K_{i}^{O}$ is in state $x_{1}^{O}$ add its number into output list: $L^{*}:=L^{*} \cup i$;

4. Incrementing the loop variable: $i:=i+1$;

5. If $i<n$, go to step 3 , otherwise the algorithm finishes.

6. Output the list of tasks available for processing.

\section{Task distribution}

The scheduling process determines a temporal order of the operations. And another tightly related process is the problem of task distribution, which comes into action when autonomous robots operate in a group. There are at least two major formulations of this problem.

The simplest method of distribution can be based on the formation of appropriate assignments, depending on the degree of usefulness or suitability of agents for carrying out certain technological operations.

In general, the suitability of an agent for a specific technological operation is determined by two key factors - the principal availability of the necessary functionality and the amount of available resources, which in a formalized form of representation takes the following form:

$$
f=\prod_{i=1}^{I} c_{i} \cdot \sum_{j=1}^{J} k_{j} \cdot r_{j},
$$

where $c_{i} \in\{0,1\}$ is a logical value, specifying the presence of the required functional capability; $r_{j}$ is an amount value of a particular resource and $k_{i}$ is a weight coefficient.

The task distribution mechanism can be described based on the matrix of agents suitability for carrying out different operations:

$$
\mathbf{F}=\left(f_{i j}\right)=\left(\begin{array}{ccc}
f_{11} & \cdots & f_{1 T} \\
\vdots & \ddots & \vdots \\
f_{R 1} & \cdots & f_{R T}
\end{array}\right),
$$

where $i=\{1, \ldots, R\}$ is robot index and $j=\{1, \ldots, \mathrm{T}\}$ is an index of technological operation. 
The output of task distributor is defined as a subset:

$$
\mathbf{A}=\left\{i_{1}, i_{2}, \ldots, i_{R}\right\}, i_{j} \in\{1,2, \ldots, T\},
$$

where $i_{j}$ is an index of technological operation; $R$ is a number of robotic agents not occupied with tasks execution; $T$ is a number of technological operations available for execution.

The task distribution problem may now be defined as a search for the optimal task appointment (4) that maximizes the overall suitability of MRS agents:

$$
\mathbf{A}: f_{\Sigma}=\sum_{j=1}^{R} f(i, j) \stackrel{i \in \mathbf{A}}{\longrightarrow} \max .
$$

Thus, it is possible both to plan and distribute the technological operations among the robots at the strategic and tactical levels of MRS control correspondingly.

\section{The problem of automatic search and removal of debris}

The task of automatic debris removal is multi-stage and complex, as it involves the use of both control and information processing technologies. The scheme of the experiment on verification of the proposed group control algorithms is shown in Fig. 2. The solution of this problem includes the following main stages:

- moving a group of robots to the starting point;

- reconnaissance of the area and determining the coordinates of the blockage;

- moving of autonomous robots to the site of debris removal;

- elementwise removal of the blockage by autonomous robots.

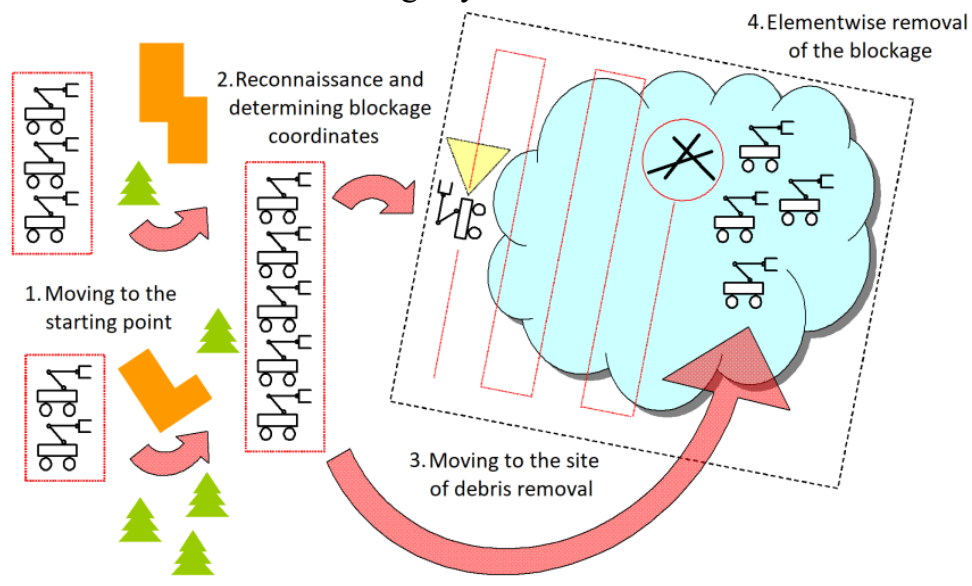

Figure 2. A scheme of the experiment on multiagent debris removal

The main requirements for the functionality of MRS are related to the need to analyze the assigned application task, decompose it into a set of subtasks or multi-stage technological operations and their subsequent fulfillment in autonomous mode by the joint efforts of individual agents coordinating their actions. The creation of such systems, fully compliant with all the technical requirements is associated with solving the following key problems:

- organization of a developed human-machine interface that allows for the rapid formulation of MRS mission;

- organization of appropriate interaction between individual agents in the interests of performing a common applied task;

- ensuring the autonomy of agents and the system as a whole. 
Adapting this concept to solve the selected problem of searching and removal of debris involves specifying the hardware and software implementation of subsystems for planning, task distribution, and information processing in the form of appropriate software modules.

Thus, Fig. 3 shows a diagram of developed software aimed at solving multi-stage applied problems using multifunctional robots that include both a mobile wheel platform and a manipulating device for capturing and transferring objects in the external environment. Such a combination of hardware and software fully meets the needs of full-scale modeling.

The principle of autonomy of robotic agents as part of MRS imposes increased requirements on their software, which should ensure the fulfillment of many tasks in conditions of environmental uncertainty. As a basis for software development, the principle of hierarchical construction of an intelligent control system for autonomous robots was taken [6]. The key functionality provided by the robot control system is goal-directed motion, area scanning motion, object detection and manipulator control to enable object grasping.

Robot navigation is based on the fusion of onboard odometry and external visual navigation data using fuzzy logic technology [7], which allows simulating GPS navigation in conditions of a closed area.

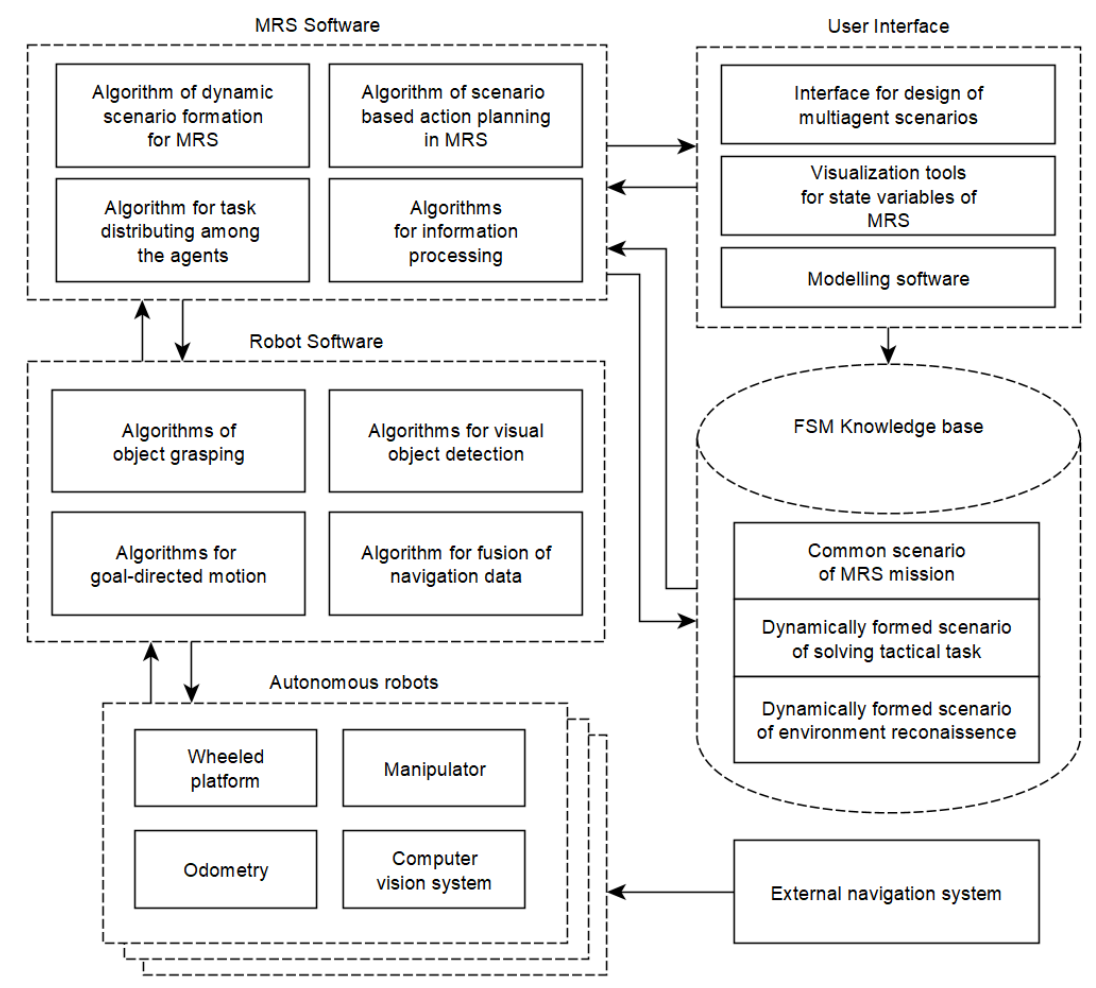

Figure 3. Block diagram of the MRS software

\section{Experiments}

Within the framework of full-scale modeling of the solution of the described problem, a robotic platform was organized on the basis of the equipment available in laboratory "Intelligent autonomous robots and multi-agent robotic systems" of RTU MIREA.

Fast setting of a multi-stage application task is achieved by describing the technological scenario by the operator. Using a computer mouse or a touch screen the expert draws a graph in 
the corresponding interface panel, the nodes of which are the generalized stages of the MRS task (Fig. 4).

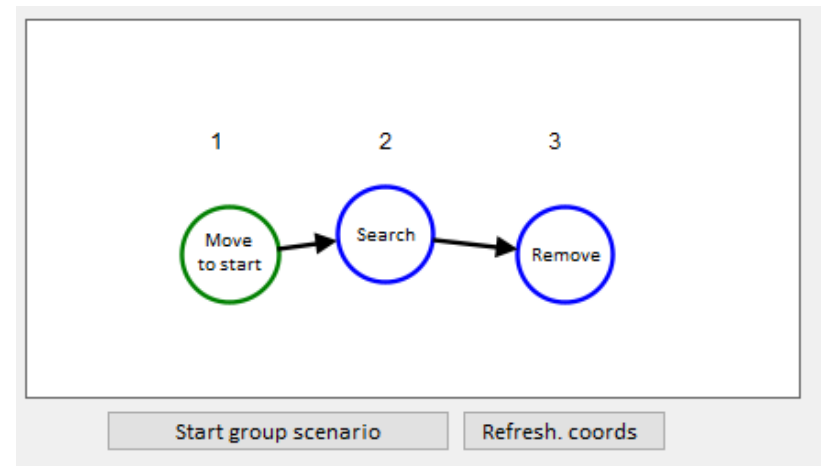

Figure 4. Top-level scenario for solving a multi-stage problem of finding and removing a blockage

We used 3 KUKA youBot platforms to verify our FSM-based control strategy [8].

After the fulfillment of the $1^{\text {st }}$ stage of the mission all robots take their places in the right side of the experimental area (Fig. 5).

During the $2^{\text {nd }}$ stage of the mission robot 1 performs area scanning using algorithms of computer vision [9] and discovers debris element O1, as it is shown in Fig. 5, a.

The $3^{\text {rd }}$ stage of the mission is related to sequential grasping and transporting of debris elements by all the robotic agents to the specified positions (Fig. 5, a-f).

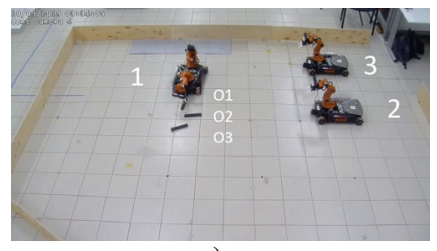

a)

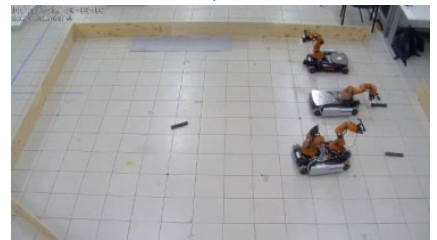

d)

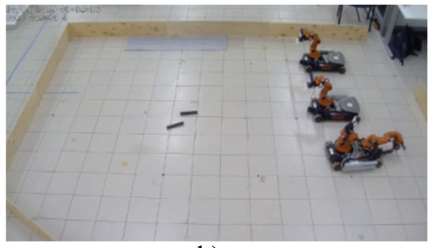

b)

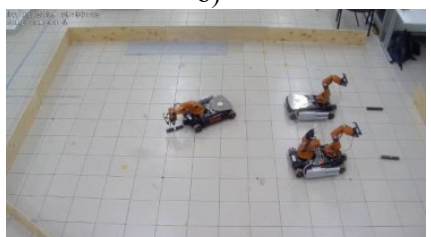

e)

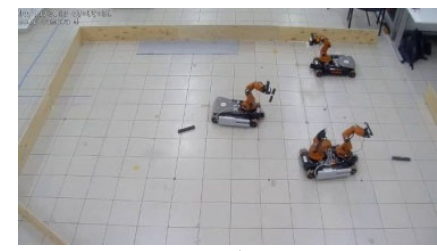

c)

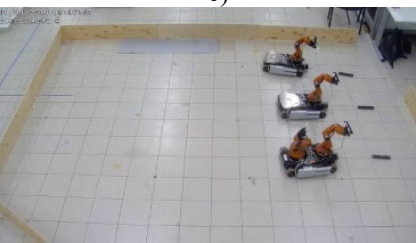

f)

Figure 5. Fragments of modeling MRS operation

It should be noted that such an experiment should account for collisions that are possible between robots sharing the same work area. Yet this problem is solved on the level of individual agent lidar data processing and route planning rather than dealing with it on the level of MRS control.

\section{Summary}

The hierarchical control of the multi-robot system allows to plan stages of a collective mission at the strategic level, distribute subtasks between robots at the tactical level and to fulfill them autonomously by the robotic agents. 
Experimental studies conducted during the presented research have proved the reality of the prospects for creating multi-agent systems based on autonomous robots for solving complex problems in conditions of uncertainty.

During the experiments, it is shown that

- due to the use of standard control elements, the efficiency of creating hierarchical scenarios for solving complex application problems is achieved;

- the technology of automatic generation of technological scenarios based on the results of analysis of sensor data received from autonomous robotic agents within MRS is applicable in practice;

- the robot's level of autonomy must correspond to the general target designations issued by the MARS planner.

This work was supported by the Russian Science Foundation under the research project No. 16-19-00052 of 2020.

\section{References}

1. I. Landa-Torres, D. Manjarres, S. Bilbao, J. Del Ser, "Underwater robot task planning using multi-objective meta-heuristics." Sensors, 2017, no. 17(4), 762.

2. S. Manko, S. Diane, V. Lokhin. "Task planning in robot groups for problems with implicitly defined scenarios based on finite-state automata technique." In Proc. of SCM-2017, SaintPetersburg, 24 - 26 May 2017.

3. Sniedovich, M. (2006). "Dijkstra's algorithm revisited: the dynamic programming connexion". Journal of Control and Cybernetics. 35 (3): 599-620.

4. H. Mohammed \& M. Jaradat \& L. Romdhane, RRT*N: An improved rapidly-exploring random tree approach for reduced processing times. 1-6. In Proc. of 2018 11th International Symposium on Mechatronics and its Applications (ISMA).

5. C. Yang and K. Y. Szeto, "Solving the Traveling Salesman Problem with a Multi-Agent System," 2019 IEEE Congress on Evolutionary Computation (CEC), Wellington, New Zealand, 2019, pp. 158-165.

6. S. Manko, S. Diane, A. Panin "Intellectual feedback in adaptive control systems" //Proceedings of the 2014 International Conference on Network Security and Communication Engineering, Hong Kong, pp. 639 - 643, 2015.

7. G. G. Kalach, Navigation System Based on the Fuzzy Logic Expert System, International Journal of Advanced Trends in Computer Science and Engineering, Volume 8, issue 6, November-December, 2019.

8. R. Bischoff, U. Huggenberger and E. Prassler, "KUKA youBot - a mobile manipulator for research and education," 2011 IEEE International Conference on Robotics and Automation, Shanghai, 2011, pp. 1-4.

9. B. Steder, R. Rusu, K. Konolige, W. Burgard. Point feature extraction on 3D range scans taking into account object boundaries. ICRA. 2601-2608. 2011. 